\title{
Immersed transparent microsphere magnifying sub-diffraction-limited objects
}

\author{
Seoungjun Lee,,${ }^{1, *}$ Lin Li, ${ }^{1}$ Zengbo Wang, ${ }^{1}$ Wei Guo, ${ }^{1}$ Yinzhou Yan, ${ }^{1}$ and Tao Wang ${ }^{2}$ \\ ${ }^{1}$ School of Mechanical, Aerospace and Civil Engineering, University of Manchester, Manchester M13 9PL, UK \\ ${ }^{2}$ Faculty of Medical and Human Science, University of Manchester, Manchester M13 9PL, UK \\ ${ }^{*}$ Corresponding author: seoungjun.lee@ postgrad.manchester.ac.uk \\ Received 28 May 2013; accepted 12 September 2013; \\ posted 25 September 2013 (Doc. ID 191245); published 15 October 2013
}

\begin{abstract}
The resolution of an optical microscope is restricted by the diffraction limit, which is approximately $200 \mathrm{~nm}$ for a white light source. We report that sub-diffraction-limited objects can be resolved in immersion liquids using a microsphere optical nanoscopy (MONS) technique. Image magnifications and resolutions were obtained experimentally and compared in different immersion liquids. We show that a $100 \mu \mathrm{m}$ diameter barium titanate $\left(\mathrm{BaTiO}_{3}\right)$ glass microsphere combined with a standard optical microscope can image sub-diffraction-limited objects with halogen light in three different media: water, $40 \%$ sugar solution, and microscope immersion oil. In this paper, the super-resolution imaging performance has been described with the three immersion liquid types and the mechanisms are discussed with Mie theory calculation in the field of a Poynting vector. (C) 2013 Optical Society of America

OCIS codes: (100.6640) Superresolution; (180.0180) Microscopy; (110.2970) Image detection systems.

http://dx.doi.org/10.1364/AO.52.007265
\end{abstract}

\section{Introduction}

Conventional optical microscopy has a theoretical limitation in optical resolution at approximately $200 \mathrm{~nm}$ in the visible spectrum because of loss of evanescent waves in the far field [1]. Imaging technologies with a sub-diffraction-limited spatial resolution are essential to nanoscience and biomedical science. One of the methods to overcome the optical diffraction limit is the use of metamaterial-based perfect superlenses operating in the near field [2]. The near-field evanescent wave could be transferred to the far field by combining the propagating wave with the evanescent wave at the image plane through the far-field optical superlens (FSL) [3-7]. However, the light source was UV or $x$ ray, and the magnification was approximately 1 . This limitation prevents the use of the technology in combination with conventional optical

$1559-128 \mathrm{X} / 13 / 307265-06 \$ 15.00 / 0$

(C) 2013 Optical Society of America microscopes because the resolution of the imaged objects is smaller than the diffraction limit of standard optical microscopes [8]. To overcome this limitation, Liu et al. demonstrated that sub-diffraction-limited objects could be magnified and observed by a conventional optical microscope with a magnifying optical hyperlens based on a curved (e.g., hypobolic) metamaterial lens, which can convert an evanescent wave into a propagating wave [9]. Nanoscale spherical lenses were used to resolve objects beyond the diffraction limit in the near field [10]. The super-oscillatory lens discovered recently achieved far-field superresolution by using an optical mask [11]. Superresolution and magnified virtual imaging were first demonstrated by Wang et al. using fused silica microspheres to image a gold-coated anodic aluminum oxide membrane with a conventional optical microscope at a record resolution of $50 \mathrm{~nm}$ [12]. Super-resolution imaging was also realized by fused silica microspheres with a semi-immersing liquid of ethanol and microscope immersion oil $[13,14]$ and a barium 


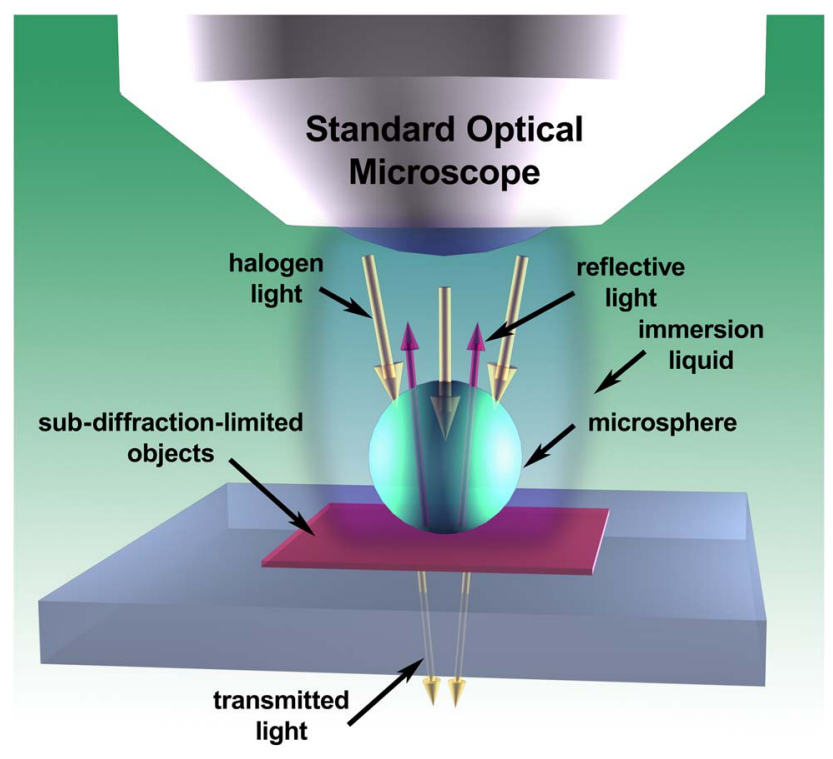

Fig. 1. Schematic of the MONS technique with a microsphere and a standard optical microscope in the reflective mode.

titanate glass microsphere immersed in isopropyl alcohol [15]. In this paper, we report the effect of different liquid immersion media (water, $40 \%$ sugar solution, and Leica microscope immersion oil) on the optical imaging performance of a $\mathrm{BaTiO}_{3}$ glass microsphere coupled with a standard optical microscope.

\section{Experimental Procedures}

The limitation on optical resolution is expressed by the Rayleigh criterion, which includes the diffraction-limited resolution $(d)$ for an optical lens with a numerical aperture (NA) at wavelength $\lambda_{0}$, such that,

$$
d=0.61 \times \frac{\lambda_{0}}{\mathrm{NA}} .
$$

Based on the equation, the resolution of standard optical microscopes can be limited to approximately $200 \mathrm{~nm}$ at visible wavelengths. Based on this, the minimum resolution of the $\times 50$ objective lens (NA: 0.75 ) is approximately $300 \mathrm{~nm}$ at the visible wavelengths. A lens with a higher NA over 1.0 may not be suitable for the microsphere optical nanoscopy (MONS) technique with large microspheres because the working distance of the lens can be smaller than the microsphere size, which results in the microsphere contacting the lens, making imaging (where the focal position needs to be below the target surface) difficult to implement. The Verbatim Blu-ray disc BD-R 25GB was examined as the sub-diffraction-limited object. The soft protect film was ejected by a shallow cut in the
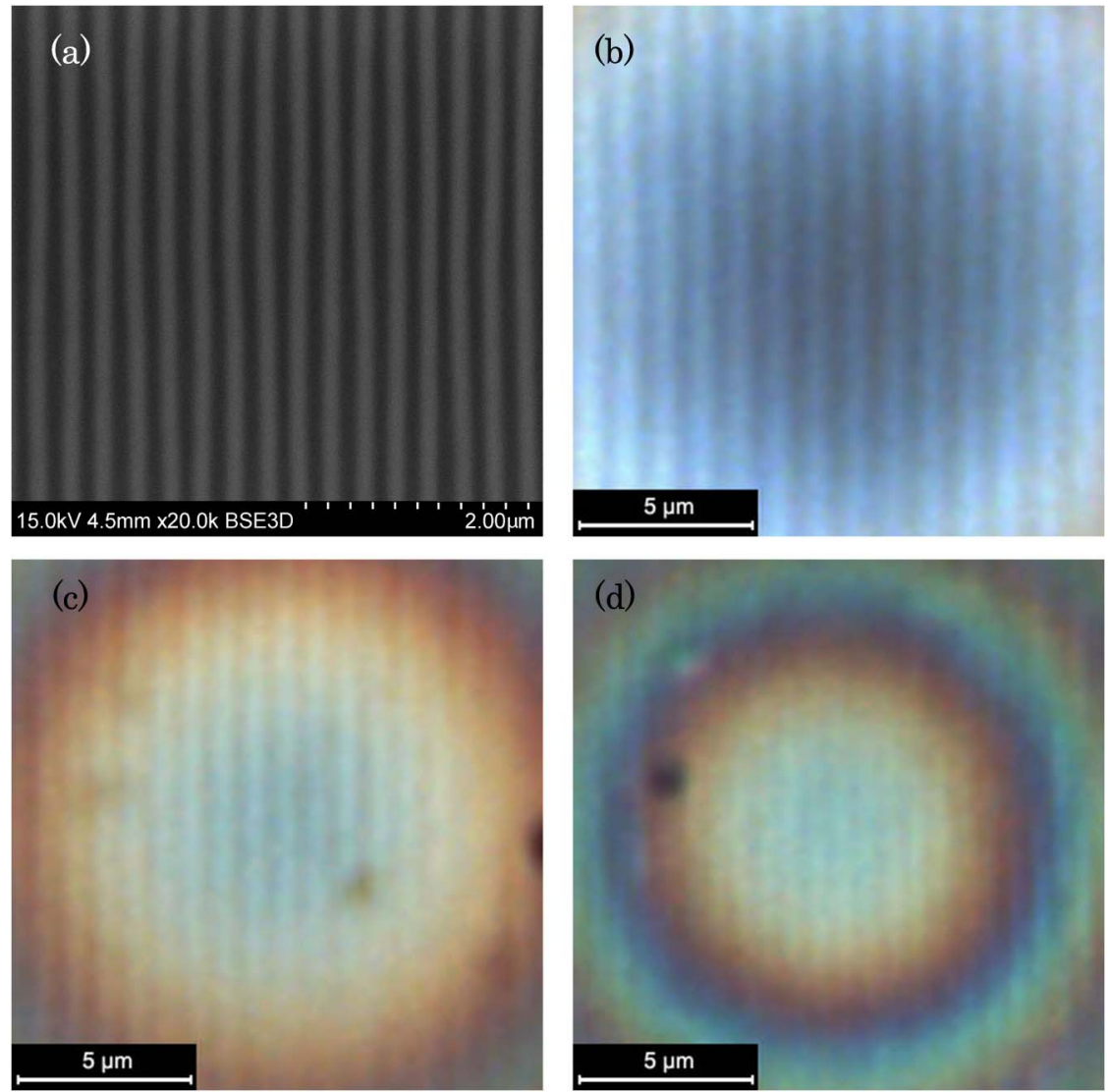

Fig. 2. Super-resolution and magnified Blu-ray disc images obtained using the MONS method in different liquid media. (a) Scanning electron microscope image of a Blu-ray disc with a line width of $120 \mathrm{~nm}$ and a spacing of $180 \mathrm{~nm}$. The magnified images through the optical microscope were observed in (b) water, (c) 40\% sugar solution, and (d) microscope immersion oil using a $100 \mu \mathrm{m}$ diameter $\mathrm{BaTiO}_{3}$ microsphere. 
Table 1. Experimentally Determined Image Magnifications and Imaging Plane Positions in the Different Media

\begin{tabular}{lccc}
\hline & Water & $\begin{array}{c}\text { 40\% Sugar } \\
\text { Solution }\end{array}$ & $\begin{array}{c}\text { Microscope } \\
\text { Immersion Oil }\end{array}$ \\
\hline Refractive index & 1.33 & 1.399 & 1.518 \\
Relative refractive & 1.429 & 1.357 & 1.252 \\
index with BaTiO & & & \\
Magnification & $3.3-3.8$ & $2.5-3.0$ & $2.0-2.5$ \\
One pitch size & $1000 \mathrm{~nm}$ & $850 \mathrm{~nm}$ & $700 \mathrm{~nm}$ \\
Groove sizes & $600 \mu \mathrm{m} /$ & $500 \mu \mathrm{m} /$ & $450 \mu \mathrm{m} /$ \\
& $400 \mu \mathrm{m}$ & $350 \mu \mathrm{m}$ & $250 \mu \mathrm{m}$ \\
Focal image position & $100 \mu \mathrm{m}$ & $62 \mu \mathrm{m}$ & $25 \mu \mathrm{m}$ \\
& below & below & below \\
Focal image range & $70-150 \mu \mathrm{m}$ & $42-87 \mu \mathrm{m}$ & $15-45 \mu \mathrm{m}$ \\
\hline
\end{tabular}

Blu-ray disc. Chemical etchings were not applied on the Blu-ray disc so that the dielectric cover layer would not be removed. The microspheres were spread on the back part of the Blu-ray disc with liquid drops. The observation of super-resolution was explored viewing the microspheres. A standard optical microscope (Leica DM $2500 \mathrm{M}$ ) was used in combination with $100 \mu \mathrm{m} \mathrm{BaTiO}_{3}$ glass microspheres immersed in water, $40 \%$ sugar solution, and Leica microscope immersion oil as shown in Fig. 1. The refractive indices of the $\mathrm{BaTiO}_{3}$ glass, water, $40 \%$ sugar solution, and Leica microscope immersion oil were 1.90 ,
$1.330,1.399$ [16], and 1.518, respectively. Halogen light was projected onto the target at the reflective mode. The field of view was adjusted to the size of the microspheres. The focal plane of the optical microscope was explored at various distances from the target surface in order to understand its effect on the image contrast and the resolution. The effects of a $100 \mu \mathrm{m} \mathrm{BaTiO}{ }_{3}$ glass microsphere immersed in the different media were examined on the imaging of the Blu-ray disc.

\section{Results and Discussion}

The Blu-ray disc lines were magnified by the $100 \mu \mathrm{m}$ $\mathrm{BaTiO}_{3}$ microsphere in three different media as shown in Fig. 2. The magnified Blu-ray disc regular lines were clearly observed in three different media with the microsphere. Without the microsphere, these lines cannot be observed. The different media generated different magnifications. The magnifications in water, $40 \%$ sugar solution, and the Leica microscope immersion oil were experimentally observed to be 3.3-3.8 times, 2.5-3.0 times, and 2.02.5 times, respectively. The 120 and $180 \mathrm{~nm}$ periodic lines of the Blu-ray disc were clearly magnified in water and in the $40 \%$ sugar solution as in Figs. 2(b) and $2(\mathrm{c})$, but the ratio of the periodic lines was not held in the Leica microscope immersion oil as in Fig. $2(\mathrm{~d})$. It may cause the magnified image to be
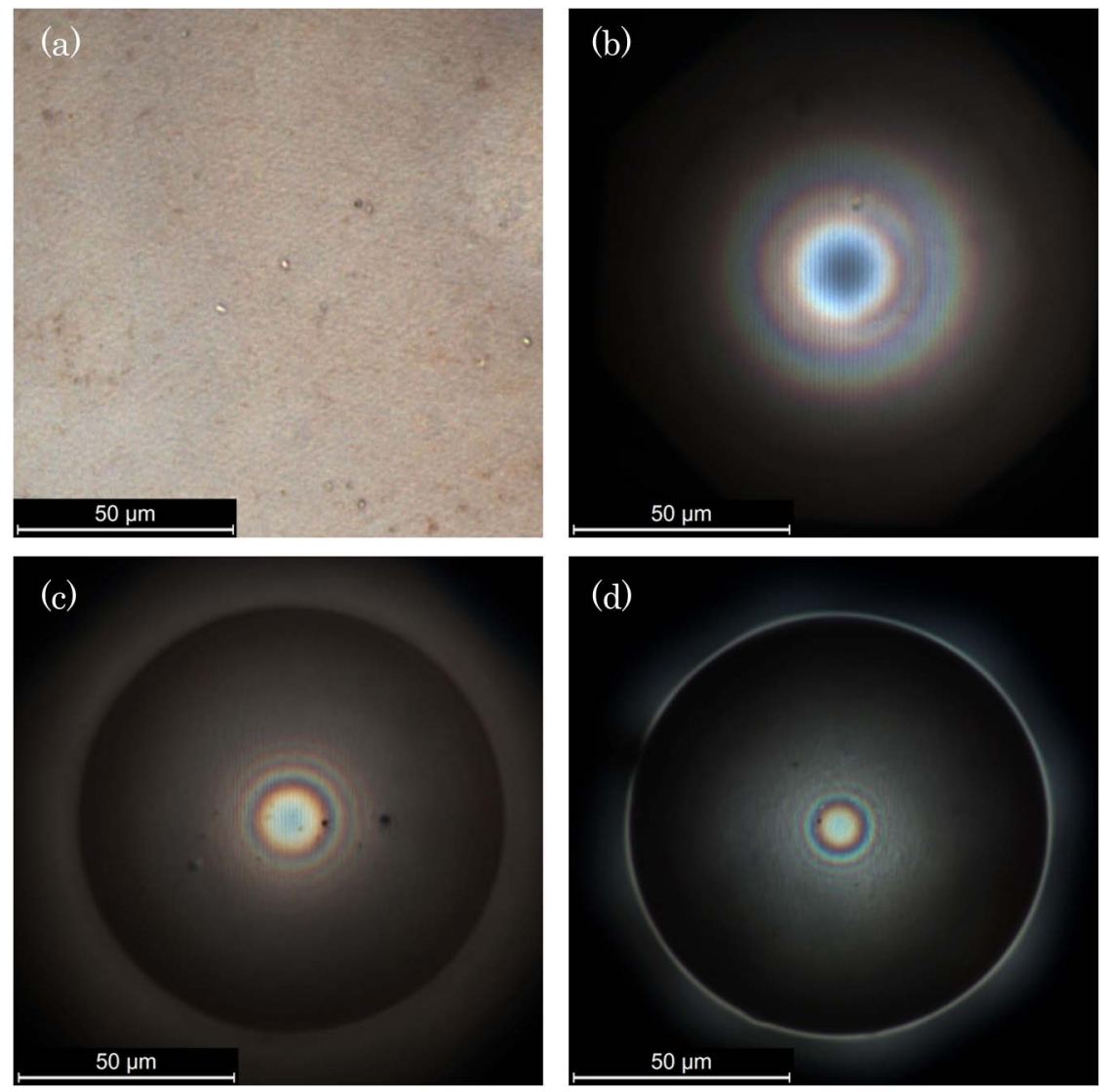

Fig. 3. Different sizes of specular highlight and magnified Blu-ray disc images in the different media. (a) Microscope optical image of the Blu-ray disc in water without the microsphere. Optical microscope images with the $100 \mu \mathrm{m} \mathrm{BaTiO}_{3}$ microsphere obtained in (b) water, (c) $40 \%$ sugar solution, and (d) Leica microscope immersion oil. 

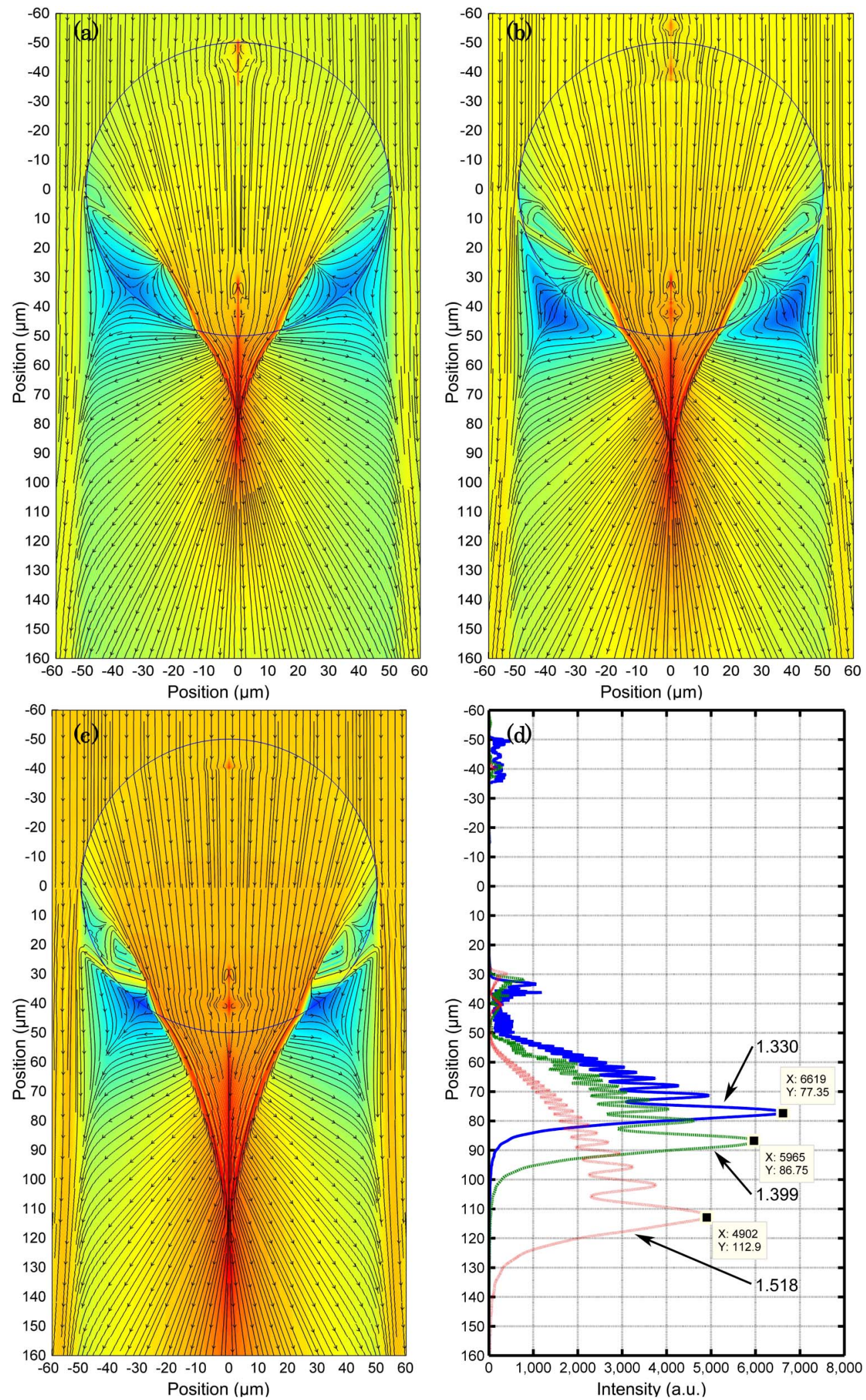

Fig. 4. Simulation of Mie theory in the field of the Poynting vector. The responses of the $100 \mu \mathrm{m} \mathrm{BaTiO}{ }_{3}$ microsphere to incident light in the three different media: (a) water, (b) $40 \%$ sugar solution, and (c) Leica microscope immersion oil. (d) Intensity distribution in the $x-z$ plane in water (solid blue, $n_{1}=1.330$ ), $40 \%$ sugar solution (dashed green, $n_{1}=1.399$ ), and Leica microscope immersion oil (short dotted red, $n_{1}=1.518$. 
smaller than the minimum resolution of the objective lens of the optical microscope, which can be limited by the NA of the object lens.

The relative refractive index, image focal positions, and magnifications for the three different media are shown in Table 1 . The relative refractive index is defined as ${ }_{1} n_{2}=\overline{n_{2}} / n_{1}$, as a function of the refractive index parameter $n_{1}$ for the medium and $n_{2}$ for the microsphere. The magnification and resolution can be affected by the relative refractive index between a $\mathrm{BaTiO}_{3}$ glass microsphere and an immersion liquid. A higher relative refractive index can increase magnification and it will enable better resolution. This is true until $n=1.8$ [12]. To use an immersion liquid, the image resolution and magnification can be decided by the relative refractive index between a microsphere and the immersion liquid rather than the refractive index of the microsphere. The immersion liquid can realize super-resolution above $n=1.8$ of the microsphere. When the image plane position was close to the target surface, the optical magnification decreased. On the other hand, when the image plane position was far below the target surface, the magnification increased. However, not all positions had good contrast in the images. The clearest and best contrast positions were generally observed close to the center of the image focal planes.

Specular highlight was observed as color rings in the focal image ranges in the three immersion liquids as shown in Fig. 3. The color rings were generated by specular reflection of the halogen light and was strongly affected by the relative refractive index between the $\mathrm{BaTiO}_{3}$ glass microsphere and the immersion liquid [17]. The size of the color rings expanded when the relative refractive index increased. The center of the blue color ring had better contrast and resolution than other color rings. In dry condition without liquid immersion, super-resolution and magnification were not realized because the windows of the superfoci could be increased; moreover, the refractive index of the $\mathrm{BaTiO}_{3}$ glass microsphere exceeds the theoretical maximum refractive index of super-resolution as up to $n=1.8$ [12].

The magnification and super-resolution of MONS can be understood by Mie theory [18]. The $100 \mu \mathrm{m}$ $\mathrm{BaTiO}_{3}$ microsphere with a parallel incoming plane wavelength of $600 \mathrm{~nm}$ is generated in the three different media as shown in Fig. 4 . The simulation was carried out using Mie theory to examine the directional streamlines and the electric intensity in the Poynting vector field. The microsphere can generate a photonic nanojet and the width of the photonic nanojet is tighter than the diffraction limit, without significant optical diffraction [19]. This optical path could be reversed in the reflective mode of a standard optical microscope so super-resolution can be observed. The maximum intensity position (MIP) can affect super-resolution imaging. When the MIP is close to the microsphere, resolution and magnification might increase. It may cause the sufficient energy to focus on the photonic nanojet. Thus, the width of the photonic nanojet might be minimized. The MIP is affected by the relative refractive index of the microsphere and the immersion liquid. A higher relative refractive index can increase the intensity and simultaneously reduce the distance between the microsphere and the MIP. The distance between the microsphere and the MIP is 27.35, 36.75 , and $62.90 \mu \mathrm{m}$ in water, $40 \%$ sugar solution, and Leica microscope immersion oil, respectively. Moreover, the distance may affect the contrast and resolution in the area of specular highlight.

\section{Conclusions}

Sub-diffraction-limited imaging using a large $\mathrm{BaTiO}_{3}$ sphere coupled with a standard optical microscope has been demonstrated in water, $40 \%$ sugar solution, and Leica microscope immersion oil. Optical magnification was approximately 3.3 times, 2.8 times, and 2.3 times in water, $40 \%$ sugar solution, and Leica microscope immersion oil, respectively. The magnified images were observed when the optical microscope was focused below the target. The focal image positions were affected by the type of immersion liquid. In water and $40 \%$ sugar solution, the magnified super-resolution image was observed clearly, but image distortion was observed in Leica microscope oil because of the limitation of the objective lens. Color rings were observed due to specular reflection through reflective halogen light and the size of the color rings could be affected by the relative refractive index between a microsphere and the immersion liquid. The super-resolution imaging can be explained by the photonic nanojet with the reversed optical path. MONS allows sub-diffraction-limited optical imaging in liquid conditions combined with standard optical microscopy. Biological samples often require liquid conditions and thus they can be observed directly without chemical processing, fluorescing, vacuum, and conducting material coating. Such potentials can facilitate the understanding of the mechanisms and the interactions of viruses, bacteria, cells, and drugs.

\section{References}

1. Y. Xiong, Z. Liu, C. Sun, and X. Zhang, "Two-dimensional imaging by far-field superlens at visible wavelengths," Nano Lett. 7, 3360-3365 (2007).

2. J. B. Pendry, "Negative refraction makes a perfect lens," Phys. Rev. Lett. 85, 3966-3969 (2000).

3. Z. Liu, N. Fang, T. J. Yen, and X. Zhang, "Rapid growth of evanescent wave by a silver superlens," Appl. Phys. Lett. 83, 5184-5186 (2003).

4. N. Fang, Z. Liu, T. J. Yen, and X. Zhang, "Regenerating evanescent waves from a silver superlens," Opt. Express 11, 682-687 (2003).

5. N. Fang, H. Lee, C. Sun, and X. Zhang, "Sub-diffractionlimited optical imaging with a silver superlens," Science 308, 534-537 (2005).

6. Z. Liu, S. Durant, H. Lee, Y. Pikus, N. Fang, Y. Xiong, C. Sun, and X. Zhang, "Far-field optical superlens," Nano Lett. 7, 403-408 (2007)

7. Z. Liu, S. Durant, H. Lee, Y. Pikus, Y. Xiong, C. Sun, and X. Zhang, "Experimental studies of far-field superlens for subdiffractional optical imaging," Opt. Express 15, 6947-6954 (2007). 
8. I. I. Smolyaninov, Y. J. Hung, and C. C. Davis, "Magnifying superlens in the visible frequency range," Science $\mathbf{3 1 5}$, 1699-1701 (2007).

9. Z. Liu, H. Lee, Y. Xiong, C. Sun, and X. Zhang, "Far-field optical hyperlens magnifying sub-diffraction-limited objects," Science 315, 1686 (2007)

10. J. Y. Lee, B. H. Hong, W. Y. Kim, S. K. Min, Y. Kim, M. V. Jouravlev, R. Bose, K. S. Kim, I. C. Hwang, L. J. Kaufman, C. W. Wong, and P. Kim, "Near-field focusing and magnification through self-assembled nanoscale spherical lenses," Nature 460, 498-501 (2009).

11. E. T. F. Rogers, J. Lindberg, T. Roy, S. Savo, J. E. Chad, M. R. Dennis, and N. I. Zheludev, "A super-oscillatory lens optical microscope for subwavelength imaging," Nat. Mater. 11, 432-435 (2012).

12. Z. Wang, W. Guo, L. Li, B. Luk'yanchuk, A. Khan, Z. Liu, Z Chen, and M. Hong, "Optical virtual imaging at $50 \mathrm{~nm}$ lateral resolution with a white-light nanoscope," Nat. Commun. 2, 1211 (2011).
13. X. Hao, C. Kuang, Y. Li, X. Liu, Y. Ku, and Y. Jiang, "Hydrophilic microsphere based mesoscopic-lens microscope (MMM)," Opt. Commun. 285, 4130-4133 (2012).

14. X. Hao, C. Kuang, X. Liu, H. Zhang, and Y. Li, "Microsphere based microscope with optical super-resolution capability," Appl. Phys. Lett. 99, 203102 (2011).

15. A. Darafsheh, G. F. Walsh, L. Dal Negro, and V. N. Astratov, "Optical super-resolution by high-index liquid-immersed microspheres," Appl. Phys. Lett. 101, 141128 (2012).

16. D. R. Lide, CRC Handbook of Chemistry and Physics, 92nd ed. (CRC Press, 2011).

17. S. Tominaga and N. Tanaka, "Refractive index estimation and color image rendering," Pattern Recogn. Lett. 24, 1703-1713 (2003).

18. Z. Wang and L. Li, "White-light microscopy could exceed $50 \mathrm{~nm}$ resolution," Laser Focus World 47, 61-64 (2011).

19. Z. Chen, A. Taflove, and V. Backman, "Photonic nanojet enhancement of backscattering of light by nanoparticles: a potential novel visible-light ultramicroscopy technique," Opt. Express 12, 1214-1220 (2004). 